\title{
Image Enhancement in Coherent Optical Amplification by Photorefractive Crystals
}

\author{
Danyu Chen, Fengchun Tian, Ying Liu, Youwen Hu, Liang Han
}

College of Communication Engineering, Chongqing University, Chongqing, China.

Email: fengchuntian@cqu.edu.cn

Received November 2013

\section{ABSTRACT}

In this paper, a model of two-wave mixing in the photorefractive crystal, which takes account of the difference in spatial frequency in a beam, has been built to study the image enhancement effect in coherent optical amplification. Based on the theoretical analysis of the model, the gain distribution for each pixel in the signal beam has been obtained. It shows that the unevenness of the gain is induced by the difference in spatial frequency in the beam. The factors that impact on the uniformity of image enhancement have been analyzed. As an example, the effects of these factors in a given photorefractive crystal have been studied through simulation.

\section{KEYWORDS}

\section{Coherent Optical Amplification; Photorefractive Effect; Image Enhancement; Unevenness of Gain}

\section{Introduction}

Coherent optical amplification, as an interesting phenomenon of great research and application value in nonlinear optics, has received growing interest in the past four decades. In a photorefractive crystal, two-wave mixing can be explained as the nonlinear interaction of intensity and phase between two incident beams through the photorefractive effect [1-3]. Plenty of effective applications of two-wave mixing have been applied to different fields, such as real-time holography, self-pumped phase conjugation, coherent image amplification, optical storage, etc. $[4,5]$.

As for two-dimensional image enhancement, it is a practical way to make use of photorefractive crystals in two-wave mixing arrangements because of the relative convenient operation and the potential for high gain [6,7]. The gain non-uniformity will introduce serious wavefront distortion and apparent polarization state degradation to the output beam. Especially the induced perturbation between the spatial intensity and phase of the beam will cause small-scale self-focusing in the beam and even devastating damage to the elements in the light path. However, most research on coherent image amplification by two-wave mixing in previous literature only emphasized on the condition of a single spatial frequency $[1,8,9]$.

In this paper, the unevenness of image enhancement in coherent optical amplification by photorefractive crystals, which takes the difference in spatial frequency in a beam into account, has been studied in detail.

\section{Theoretical Analysis}

The model of two-wave mixing in previous literatures, which are concentrated on the situation for a single spatial frequency, has been presented in Figure 1.

As seen in Figure 1, the two beams $I_{1}$ and $I_{2}$ enter into a photorefractive crystal symmetrically. The interference intensity $I(x)$ and refractive index $n(x)$ distributing Equations [6] can be expressed as

$$
\begin{gathered}
I(x)=I_{0}(1+m \cos k x), \\
n(x)=n_{0}+m \Delta n_{s} \cos (k x+\psi),
\end{gathered}
$$

where $m=\frac{2 \sqrt{I_{1} I_{2}}}{I_{1}+I_{2}}$ is the intensity modulation depth; $\Delta n_{s}$ is the saturation value of photo induced refractive index change; $k$ is the value of index gratings wave vector; $\psi$ is the phase offset between the interference intensity pattern and refractive index gratings.

In order to study the unevenness of gain in coherent optical amplification by photorefractive crystals, a model that takes account of the difference in spatial frequency in a beam has been built. And the geometrical configuration has been presented in Figure 2. 


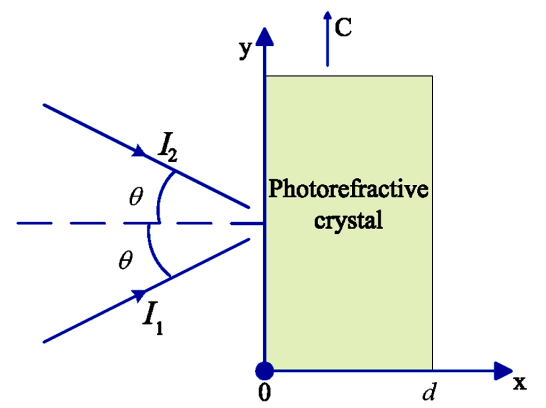

Figure 1. Previous model of two-wave mixing. This is a geometrical configuration with regard to a single spatial frequency.

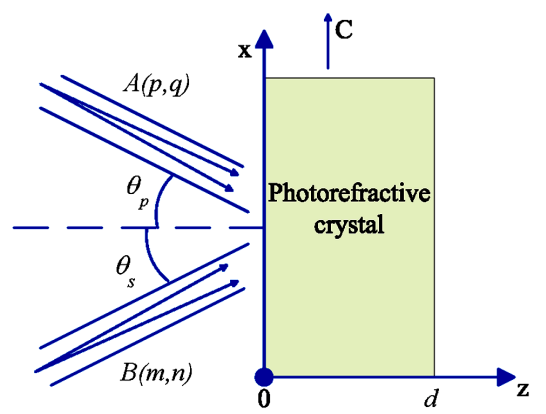

Figure 2. New model of two-wave mixing. This is a geometrical configuration in view of the different spatial frequencies in a beam.

As seen in Figure 2, C is the optical axis of the crystal, which is parallel to $\mathrm{x}$-axis. Assuming the pump and signal beam enter into the medium symmetrically with respect to the normal (z-axis) from the left side $(z=0)$. The two beams couple with each other in the crystal, which leads to the enhancement of the signal beam at the expense of the pump beam intensity decrease. For both the pump and signal beam, which are superposition of cracks of light, the optical field analytical expression on each pixel can be represented by $\mathbf{A}(p, q) \exp [\mathrm{i}(\omega t-\mathbf{a}(p, q) \cdot \mathbf{r})]$ and $\mathbf{B}(m, n) \exp [\mathrm{i}(\omega t-\mathbf{b}(m, n) \cdot \mathbf{r})]$ separately, where $(p, q)$ indicates the spatial locations of light on individual pixel in the pump beam, and $(m, n)$ similarly in the signal beam; $p=1,2, \ldots, P ; q=1,2, \ldots, Q ; m=1,2, \ldots, M ; n$ $=1,2, \ldots, N ; \mathbf{a}(p, q)$ and $\mathbf{b}(m, n)$ are separately the wave vectors on each pixel in the pump and signal beam, which can be expressed with the coordinate system in Figure 2.

As a small divergence angle will be induced with the spatial propagation in Gaussian beam shot from a laser [10], the pump and signal waves span a rather small angular range, which results in different spatial frequencies in a beam. The offsets of the propagation direction on each pixel to the average propagation direction are represented by $\delta_{p}(p, q)$ and $\delta_{s}(m, n)$ separately for the pump and signal waves. Therefore, when entering into the crystal, the incident angle on each pixel in the pump and signal beam can be represented separately by $\alpha(p, q)=\theta_{p}+\delta_{p}(p, q) \quad$ and $\quad \beta(m, n)=\theta_{s}+\delta_{s}(m, n)$, where $\theta_{p}$ and $\theta_{s}$ indicate the average incident angle of the pump and signal beam, respectively. Ordinarily, $\theta_{s}=\theta_{p}=\theta$ is assumed.

As is known, the far-field small divergence angle $\delta$ of laser beams can be figured out by the formula [11]

$$
2 \delta=\frac{2 \lambda}{\pi \omega_{0}}, \omega_{0}=\sqrt{\frac{\lambda L}{2 \pi}},
$$

where $L$ is the resonant cavity length of the laser, $\lambda$ is the wavelength of the beams. Consequently, for the pump and signal waves, $\delta_{p}(p, q)$ and $\delta_{s}(m, n)$ vary over a range of $\pm \delta$ separately, i.e., the incident angle on each pixel in the signal beam $\beta(m, n)$ range from $\theta-\delta$ to $\theta+\delta$.

The interference intensity of the pump and signal waves in the crystal, which has been deduced from Equation (1), can be expressed as

$$
\begin{aligned}
& I=I_{0}+\left(\frac{1}{2} \sum_{p=1}^{P} \sum_{q=1}^{Q} A(p, q) \sum_{m=1}^{M} \sum_{n=1}^{N} B^{*}(m, n) \exp [\mathrm{i}(\mathbf{a}(p, q)-\mathbf{b}(m, n)) \cdot \mathbf{r}]\right. \\
& \left.+\frac{1}{4} \sum_{\substack{p=1 \\
p \neq x}}^{P} \sum_{\substack{q=1 \\
q \neq y}}^{Q} A(p, q) \sum_{x=1}^{P} \sum_{y=1}^{Q} A^{*}(x, y) \operatorname{ex} \operatorname{pi}(\mathbf{a}(p, q)-\mathbf{a}(x, y)) \cdot \mathbf{r}\right] \\
& \left.\left.+\frac{1}{4} \sum_{\substack{m=1 \\
m \neq j}}^{M} \sum_{n=k}^{N} B(m, n) \sum_{j=1}^{M} \sum_{k=1}^{N} B^{*}(j, k) \operatorname{ex} \operatorname{pi}(\mathbf{b}(m, n)-\mathbf{b}(j, k)) \cdot \mathbf{r}\right]\right)+ \text { c.c. }
\end{aligned}
$$

where $I_{0}=\sum_{p=1}^{P} \sum_{q=1}^{Q} I_{p}(p, q ; 0)+\sum_{m=1}^{M} \sum_{n=1}^{N} I_{s}(m, n ; 0)$ is the total intensity of the incident pump and signal beam; c.c. indicates the complex conjugate of the previous term.

Based on Equation (2), the refractive index distribution can then be approximated as

$$
\begin{aligned}
n & =n_{0}+\mathrm{i} \frac{n_{1}}{2 I_{0}} \sum_{p=1}^{P} \sum_{q=1}^{Q} A(p, q) \sum_{m=1}^{M} \sum_{n=1}^{N} B^{*}(m, n) \text { ex }[\mathrm{pi}(\mathbf{a}(p, q)-\mathbf{b}(m, n)) \cdot \mathbf{r}] \\
& + \text { c.c. }
\end{aligned}
$$

where $n_{0}$ is the refractive index of the crystal when no light is present; $n_{1}$ is the modulation factor of refractive index, which depends on the spacing and direction of the grating, as well as on the material properties of the crystal, e.g., the electro-optic coefficient. Equation (5) is solved for the steady state so that the amplitudes $A(p, q)$ and $B(m, n)$ are taken to be time independent.

With this and the slowly varying amplitude approximation [12], Maxwell's scalar equations yield the following coupled amplitude equations

$$
\begin{gathered}
\frac{\mathrm{d} A(p, q ; r)}{\mathrm{d} r}=-\frac{\gamma_{p}(p, q)}{I_{0}} \sum_{m=1}^{M} \sum_{n=1}^{N}|B(m, n ; r)|^{2} A(p, q ; r)-\frac{1}{2} \sigma A(p, q ; r) \\
p=1,2, \ldots, P ; q=1,2, \ldots, Q ; \gamma_{p}(p, q)=\frac{\pi n_{1}}{\lambda \cos \alpha(p, q)}
\end{gathered}
$$




$$
\begin{gathered}
\frac{\mathrm{d} B(m, n ; r)}{\mathrm{d} r}=\frac{\gamma_{s}(m, n)}{I_{0}} \sum_{p=1}^{P} \sum_{q=1}^{Q}|A(p, q ; r)|^{2} B(m, n ; r)-\frac{1}{2} \sigma B(m, n ; r) \\
m=1,2, \ldots, M ; n=1,2, \ldots, N ; \gamma_{\mathrm{s}}(m, n)=\frac{\pi n_{1}}{\lambda \cos \beta(m, n)}
\end{gathered}
$$

where $\sigma$ is the absorption coefficient of the crystal, which is regulated by the wavelength of the incident beam.

Since the relation between the intensities and amplitudes of the incident beams are $I_{p}(p, q ; r)=|A(p, q ; r)|^{2}$ and $I_{s}(m, n ; r)=|B(m, n ; r)|^{2}$, the coupled intensity equations are given by

$$
\begin{gathered}
\frac{\mathrm{d} I_{p}(p, q ; r)}{\mathrm{d} r}=-\frac{\Gamma_{p}(p, q)}{I_{0}} \sum_{m=1}^{M} \sum_{n=1}^{N} I_{s}(m, n ; r) I_{p}(p, q ; r)-\sigma I_{p}(p, q ; r) \\
p=1,2, \ldots, P ; q=1,2, \ldots, Q ; \Gamma_{p}(p, q)=2 \gamma_{p}(p, q)
\end{gathered}
$$

$$
\begin{gathered}
\frac{\mathrm{d} I_{s}(m, n ; r)}{\mathrm{d} r}=\frac{\Gamma_{s}(m, n)}{I_{0}} \sum_{p=1}^{P} \sum_{q=1}^{Q} I_{p}(p, q ; r) I_{s}(m, n ; r)-\sigma I_{s}(m, n ; r) \\
m=1,2, \ldots, M ; n=1,2, \ldots, N ; \Gamma_{s}(m, n)=2 \gamma_{s}(m, n)
\end{gathered}
$$

In the assumed diffusion driven scenario, the phases of the beams are decoupled so that the intensity Equations (8) and (9) describe the two-wave mixing process completely.

With Equation (8) and Equation (9), one can integrate to yield the intensity versus effectively acting distance $r$ on each pixel in the signal beam, which can be normalized by the initial intensity as

$$
\frac{I_{s}(m, n ; r)}{I_{s}(m, n ; 0)}=\frac{1+h}{1+h e^{-\Gamma_{s}(m, n) r}} e^{-\sigma r}, h=\frac{\sum_{p=1}^{P} \sum_{q=1}^{Q} I_{p}(p, q ; 0)}{\sum_{m=1}^{M} \sum_{n=1}^{N} I_{s}(m, n ; 0)}
$$

Therefore, the gain, which is defined as the intensity ratio of the output signal wave in the presence of a pump beam to that in the absence of a pump beam, can be given by

$$
\begin{aligned}
& G(m, n)=\frac{I_{s}(m, n ; l)}{I_{s}(m, n ; 0)}=\frac{1+h}{1+h e^{-\Gamma_{s}(m, n) l}} e^{-\sigma l}, \\
& l=\frac{d}{\cos \beta_{i}(m, n)}
\end{aligned},
$$

where $d$ is the thickness of the crystal, $\beta_{i}(m, n)$ is the angle between the propagation direction and the normal (z-axis) on each pixel in the signal beam inside the crystal, and $l$ is the effective interaction length on individual pixel in the signal beam.

To perform calculation of the gain, the expression of intensity coupling coefficient $\Gamma_{s}(m, n)$ [13], presented below, can be insert into Equation (11).

$$
\Gamma_{s}(m, n)=\frac{A \sin \beta(m, n)}{1+B^{-2} \sin ^{2} \beta(m, n)} \cdot \frac{\cos 2 \beta_{i}(m, n)}{\cos \beta_{i}(m, n)} .
$$

In Equation (12), the related parameters are included in coefficient $A$ and $B$, which can be given by

$$
\begin{gathered}
A=\gamma_{\text {eff }} \xi(K) \frac{8 \pi^{2} n^{3} k_{B} T}{e \lambda^{2}}, \\
B=\left(\frac{N_{e f f}}{\varepsilon \varepsilon_{0} k_{B} T}\right)^{1 / 2} \cdot \frac{e \lambda}{4 \pi},
\end{gathered}
$$

where $\gamma_{\text {eff }}$ is the effective electric-optic coefficient; $n$ is the refractive index; $\xi(K)$ is the recombination constant, which is fixed when the value of $k=2 \pi / \lambda$ is set; $N_{\text {eff }}$ is the effective charge density, which is regulated by the wavelength of the incident beams; $k_{B}$ is the Boltzmann constant; $T$ is the absolute temperature; $e$ is the charge on the electron; $\lambda$ is the wavelength of the incident beams.

According to the analysis above, the gain non- uniformity in the signal beam, which indicates the unevenness for image enhancement, is induced by the difference in spatial frequency in the beam. As seen in Equations (11-14), the factors, which impact on the unevenness of gain in the signal beam, are the crystal thickness $d$, the incident angle $\beta(m, n)$, and the wavelength $\lambda$.

\section{Simulation and Discussion}

The non-uniformity of gain can be measured with the relative standard deviation $\gamma=S D / A V G$. Here, $A V G$ and $S D$ are the mean value and standard deviation of the gain in the signal image beam, respectively. The gain non-uniformity is negligible compared with the value of gain when $\gamma$ reaches a low enough value. The usual requirement is $\gamma=1 \%$.

In this paper, the numerical simulation for two-wave mixing is based on the model analyzed above. Assuming the signal beam and the pump beam are split from a laser beam at a ratio of $1: 1120$. Then the two of them recombines at a photorefractive

$\mathrm{Fe}(0.04 \mathrm{wt} \%): \mathrm{Ce}(0.1 \mathrm{wt} \%): \mathrm{LiNbO}_{3}$ crystal from the same side with the average incident angle $\theta_{s}=\theta_{p}=\theta$. Three scenarios with different wavelengths have been analyzed to obtain the gain distribution in the signal image beam.

\subsection{Argon Laser at $\lambda=488 \mathrm{~nm}$}

In the first scenario, the light source is an argon laser with a cavity length of $L=150 \mathrm{~cm}$ operating at wavelength $488 \mathrm{~nm}$, in which case the crystal has the parameters of $\sigma=21.2 \mathrm{~cm}^{-1}$ and $N_{\text {eff }}=2.9 \times 10^{15} \mathrm{~cm}^{-3}$ according to [14]. Substituting $\lambda$ and $L$ into Equation (3) yields the far-field small divergence angle of the laser 
beam: $\delta=0.026075^{\circ}$.

Computer-generated plots of the non-uniformity of gain versus the average incident angle $\theta$ for different values of $d$ have been presented in Figure 3. The effects of angle $\theta$ on $\gamma$ are similar for different values of $d$. $\gamma$ decreases with the increasing $\theta$, reaches a minimum, then increases and afterwards decreases again. For each value of $d, \quad \gamma$ reaches its minimum with a same value of $\theta$, i.e., $17^{\circ}$.

The values of $A V G, S D$ and $\gamma$ versus $d$ for the optimal average incident angle $\theta_{o p t}=17^{\circ}$ are presented in Figure 4. Within the $0-0.33 \mathrm{~cm}$ range of $d, \gamma$ increases with the increasing $d$, then decreases to a minimum and afterwards increases again. Particularly, the optimal thickness of the crystal $d_{o p t}$, in which case $\gamma$ reaches its minimum $0.00016 \%$, is turned out to be $0.17 \mathrm{~cm}$.

\subsection{Argon Laser at $\lambda=514.5 \mathrm{~nm}$}

The second scenario is that, the operating wavelength of the argon laser used in the first scenario is changed to $514.5 \mathrm{~nm}$, in case the crystal has the parameters of $\sigma=16.2 \mathrm{~cm}^{-1}$ and $N_{\text {eff }}=1.9 \times 10^{15} \mathrm{~cm}^{-3}$ according to [14]. What's more, the far-field small divergence angle of the laser beam is turned into $\delta=0.026774^{\circ}$.

The values of $\gamma$ versus angle $\theta$ for different values of $d$ have been presented in Figure 5. The optimal average incident angle $\theta_{\text {opt }}$, which will guarantee the minimum value of $\gamma$ regardless of the value of $d$, is appeared to be $15^{\circ}$.

Simulation results of $A V G, S D$ and $\gamma$ versus $d$ for $\theta_{o p t}$ are presented in Figure 6. Within the $0-0.43 \mathrm{~cm}$ range of $d, \gamma$ increases with the increasing $d$, then basically remain unchanged.

\subsection{He-Ne Laser at $\lambda=632.8 \mathrm{~nm}$}

The last scenario is that, the argon laser used in the first scenario is changed for a He-Ne laser with a cavity length of $L=30 \mathrm{~cm}$ at wavelength $632.8 \mathrm{~nm}$, in which case the crystal has the parameters of $\sigma=0.7 \mathrm{~cm}^{-1}$ and $N_{\text {eff }}=0.1 \times 10^{15} \mathrm{~cm}^{-3}$. Besides, the far-field small divergence angle of the laser beam is turned out to be $\delta=0.066395^{\circ}$.

The results of $\gamma$ with varying angle $\theta$ for different values of $d$ are presented in Figure 7. Note that the fluctuation amplitude of $\gamma$ is larger than that in the previous two scenarios. The optimal average incident angle $\theta_{\text {opt }}$ is $4^{\circ}$.

The values of $A V G, S D$ and $\gamma$ versus $d$ for $\theta_{\text {opt }}$ have been presented in Figure 8. $\gamma$ increases with the increasing $d$, then decreases to a minimum and afterwards increases again. The minimum value of $\gamma$ $(0.000184 \%)$ can be reached with $d_{\text {opt }}=0.54 \mathrm{~cm}$.

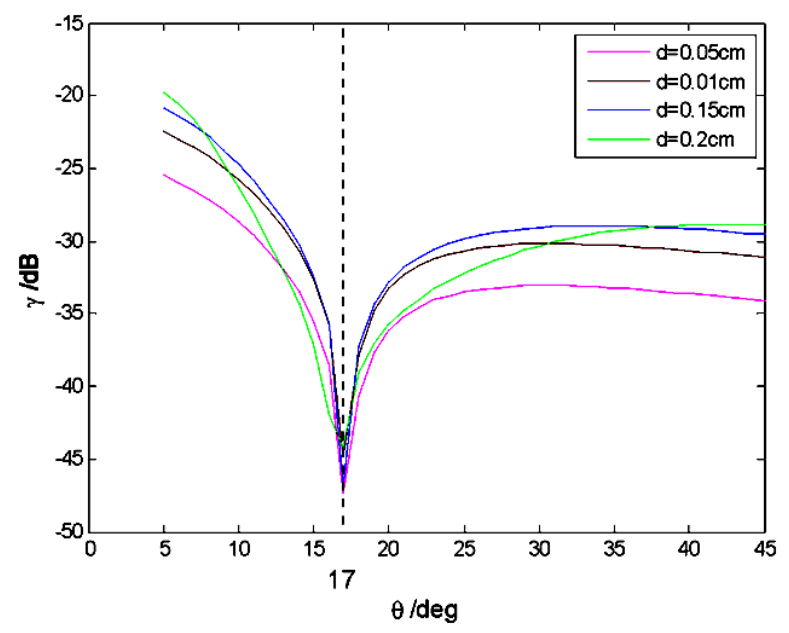

Figure 3. Values of $\gamma$ versus average incident angle $\theta$ for several values of crystal thickness $d$ with $\lambda=488 \mathrm{~nm}$.

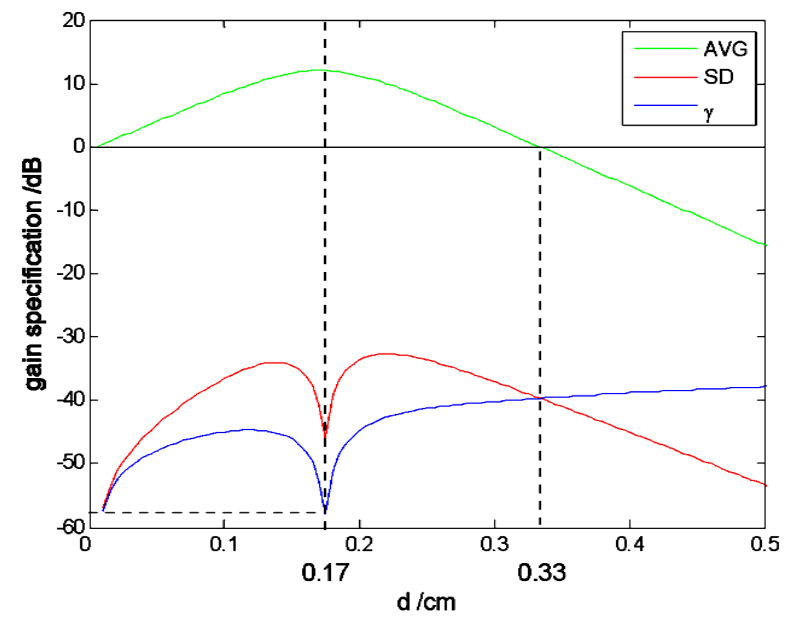

Figure 4. Values of $A V G, S D$ and $\gamma$ versus crystal thickness $d$ for optimal average incident angle $\theta_{\text {opt }}=17^{\circ}$ with $\lambda=$ $488 \mathrm{~nm}$.

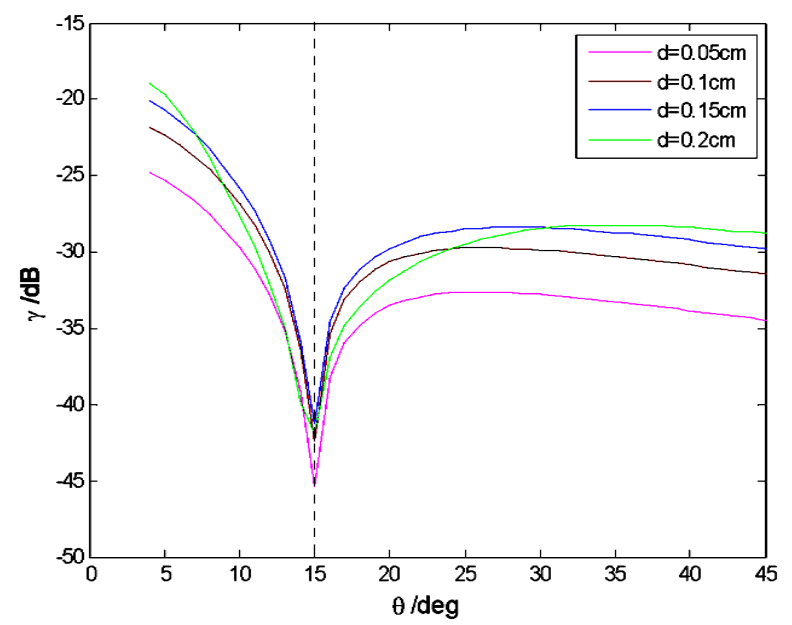

Figure 5. Values of $\gamma$ versus average incident angle $\theta$ for several values of crystal thickness $d$ with $\lambda=514.5 \mathrm{~nm}$. 


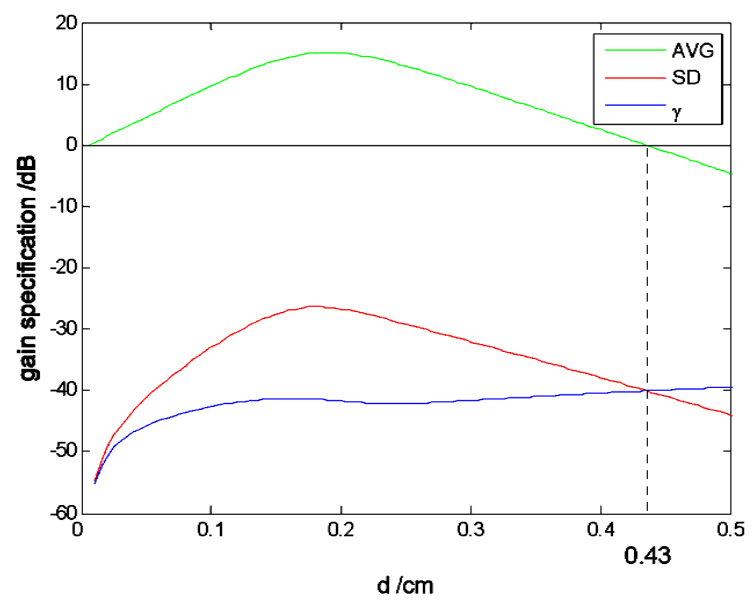

Figure 6. Values of $A V G, S D$ and $\gamma$ versus crystal thickness $d$ for optimal average incident angle $\theta_{\text {opt }}=15^{\circ}$ with $\lambda=$ $514.5 \mathrm{~nm}$.

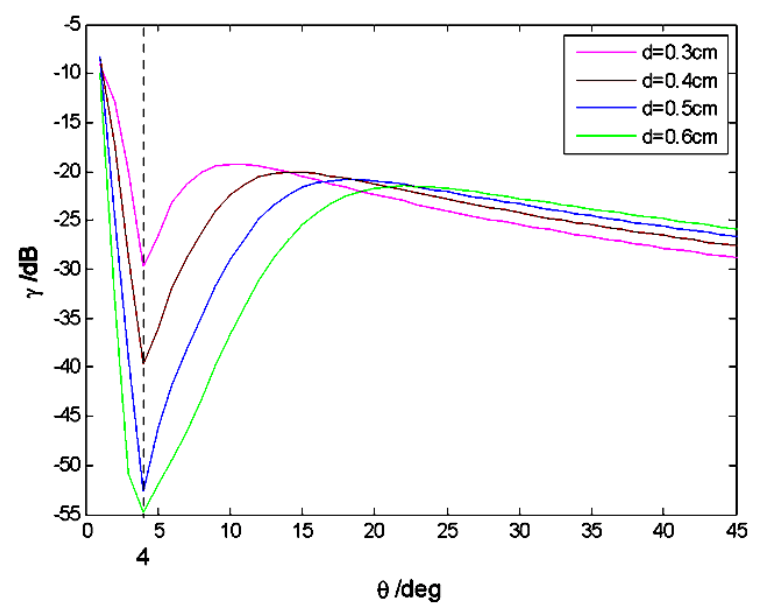

Figure 7. Values of $\gamma$ versus average incident angle $\theta$ for several values of crystal thickness $d$ with $\lambda=632.8 \mathrm{~nm}$.

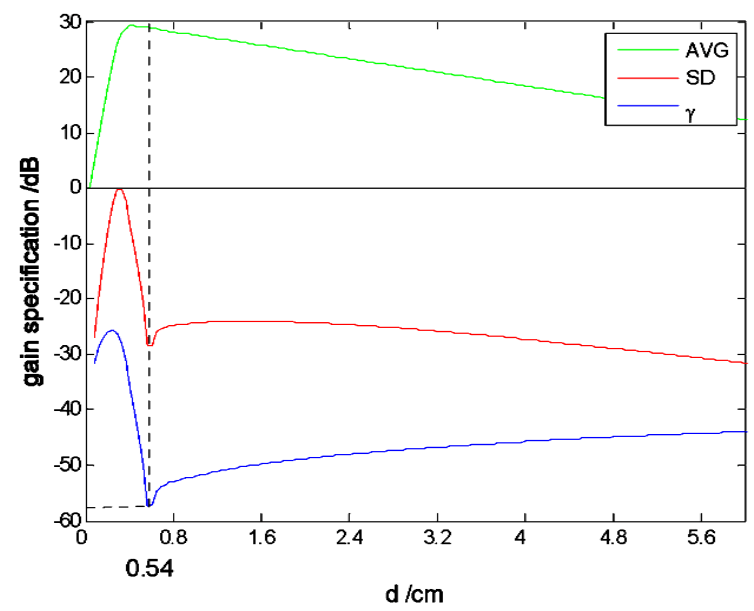

Figure 8. Values of $A V G, S D$ and $\gamma$ versus crystal thickness $d$ for optimal average incident angle $\theta_{\text {opt }}=4^{\circ}$ with $\lambda=$ $632.8 \mathrm{~nm}$.

\section{Conclusion}

In short, to explore the unevenness of image enhancement in coherent optical amplification by photorefractive crystals, a model in view of different spatial frequency in a beam has been built. It has been proved that the factors which impact on the unevenness of image enhancement are: the thickness of photorefractive crystal, the incident angle on each pixel in signal image beam, and the wavelength of incident beams. As an example, the effects of these factors in photorefractive

$\mathrm{Fe}(0.04 \mathrm{wt} \%): \mathrm{Ce}(0.1 \mathrm{wt} \%): \mathrm{LiNbO}_{3}$ crystal have been re- searched through simulation. It turns out that a considerable non-uniformity of $\gamma=0.00016 \%$ can be reached when the wavelength of the incident beam is 488 nm (at $\left.\theta=17^{\circ}, d=0.17 \mathrm{~cm}\right)$.

\section{Acknowledgements}

The authors thank the National Natural Science Foundation of China (No. 61071190, No. 61171158) and the Fundamental Research Funds for the Central Universities of China (No. CDJZR12160010) for support.

\section{REFERENCES}

[1] N. V. Kukhrarev, V. B. Markov, S. G. Odulov, M. S. Soskin and V. L. Vinetskii, "Holographic Storage in Electro-Optic Crystals. I. Steady State”, Ferroelectrics, Vol. 22, No. 1, 1978, pp. 949-953. http://dx.doi.org/10.1080/00150197908239450

[2] M. C. Søren, B. Jensen, J. P. Huignard and P. M. Petersen, "Two-Wave Mixing in a Broad-Area Semiconductor Amplifier," Optics Express, Vol. 14, No. 25, 2006, pp. 1237312379. http://dx.doi.org/10.1364/OE.14.012373

[3] M. C. Søren, B. Jensen, J. P. Huignard and P. M. Petersen, "Nonlinear Gain Amplification Due to Two-Wave Mixing in a Broad-Area Semiconductor Amplifier with Moving Gratings," Optics Express, Vol. 16, No. 8, 2008, pp. 5565-5571. http://dx.doi.org/10.1364/OE.16.005565

[4] P. Günter and J. P. Huignard, "Photorefractive Materials and Their Applications II,” Springer-Verlag, Berlin, 1989.

[5] N. Katyal, Natasha, A. Roy and A. Kapoor, "The Influence of Pump Beam Polarization on the Signal Beam, in TWM in Photorefractive Crystals: Transmission Geometry,” Optik, Vol. 122, No. 3, 2011, pp. 207-210. http://dx.doi.org/10.1016/j.ijleo.2009.11.028

[6] J. P. Huignard and A. Marrakchi, "Coherent Signal Beam Amplification in Two-Wave Mixing Experiments with Photorefractive $\mathrm{Bi}_{12} \mathrm{SiO}_{20}$ Crystals," Optics Communications, Vol. 38, No. 4, 1981, pp. 249-254. http://dx.doi.org/10.1016/0030-4018(81)90392-8

[7] Y. Fainman, E. Klancnik and S. H. Lee, “Optimal Coherent Image Amplification by Two-Wave Coupling in Photorefractive $\mathrm{BaTiO}_{3}$," Optical Engineering, Vol. 25, No. 2, 1986, pp. 228-234. http://dx.doi.org/10.1117/12.7973810 
[8] P. Tayebati and D. Mahgerefteh, "Theory of the photorefractive effect for $\mathrm{Bi}_{12} \mathrm{SiO}_{20}$ and $\mathrm{BaTiO}_{3}$ with shallow traps," Journal of the Optical Society of America B, Vol. 8, No. 5, 1991, pp. 1053-1064. http://dx.doi.org/10.1364/JOSAB.8.001053

[9] B. G. Peng, F. Chen, Y. Tan and D. Kip, “Two-Wave Mixing of Ion-Implanted Photorefractive Waveguides in Near-Stoichiometric Fe:LiNbO 3 Crystals,” Optics Materials, Vol. 33, No. 6, 2011, pp. 773-776. http://dx.doi.org/10.1016/j.optmat.2010.12.016

[10] Jiabi Chen, "Principle and Application of Laser,” Tongping Han, Beijing, 2004.

[11] D. Z. Anderson and R. Saxena, "Theory of Multimode Operation of a Unidirectional Ring Oscillator Having Photorefractive Gain: Weak Field Limit," Journal of the
Optical Society of America B, Vol. 4, No. 2, 1987, pp. 164-176. http://dx.doi.org/10.1364/JOSAB.4.000164

[12] Z. Y. Li, B. Y. Gu and G. Z. Yang, "Slowly Varying Amplitude Approximation Appraised by Transfer-Matrix Approach,” Physical Review B, Vol. 60, No. 15, 1999, pp. 10644-10647.

http://dx.doi.org/10.1103/PhysRevB.60.10644

[13] C. H. Yang and L. Sun, "Photorefractive Nonliear Optical Materials: $\mathrm{LiNbO}_{3}$ Crystals,” Jiafu Yang, Beijing, 2009.

[14] T. K. Yadav, M. K. Maurya and R. A. Yadav, "Effect of Photoconductivity and Oscillation Frequency Shift on the Signal Beam Intensity in Two Beam Coupling in Photorefractive Materials,” Optik, Vol. 122, No. 18, 2011, pp. 1607-1614. http://dx.doi.org/10.1016/j.ijleo.2010.10.011 